\title{
THE VOLUME OF WOOD FOREST RESOURCES IN THE EUROPEAN UNION COUNTRIES
}

Anna TURCZAK, The West Pomeranian Business School in Szczecin, Faculty of Economics and Computer Science, 53 Żołnierska Street, 71-210 Szczecin, Poland, aturczak@zpsb.pl

\begin{abstract}
The contributions of forests to the well-being of humankind are extraordinarily vast and far-reaching. They are an important element in mitigating climate change. The aim of the paper is to determine the influence of particular factors on the diversity of the European Union countries in terms of the amount of wood forest resources compared with the country size. Two factors affecting the variable have been analysed in the paper: 1) the growing stock per 1 hectare of forest area and 2) the quotient of the forest area and the land area without inland water. Those two independent variables are directly proportional to the dependent variable, thus the higher the growing stock density and the higher the forest cover, the bigger the amount of wood forest resources of the analysed country. The causal analysis allowed to answer the question how the two factors affect the variable considered in the twenty eight countries, namely, what the direction and the strength of their influence are. The logarithmic method was used to carry out the causal analysis. The average results obtained for the entire European Union were compared with those received for each country separately and, on this basis, final conclusions were drawn. Data for 2005, 2010 and 2015 have been used for all needed calculations.
\end{abstract}

Keywords: forest cover, forest growing stock, the European Union, wood forest resources.

\section{INTRODUCTION}

The key term in political sciences, public administration and management sciences for the last few decades has been governance (Bevir, 2010; Held and McGrew, 2002; Hooghe and Marks, 2001; Pierre, 2000; Pierre and Peters, 2000). Etymologically, the word can be traced back to the Greek verb 'kubernan', which means 'to pilot' or 'to steer' (Kjaer, 2004). Within a short time, forest governance has become a very popular concept, both among scientists and practitioners (Arts et al., 2012). In its broadest sense the concept refers to governing or steering society towards sustainable forest management by whatever institutions, but the most common interpretation is of new modes of governing forest issues that go beyond traditional government, such as policy networks, certification schemes, social corporate responsibility, participatory forest management, markets for ecosystem services, public-private partnerships and the like (Arts and Visseren-Hamakers, 2012). The field is therefore extensive and complex.

Forest governance is also gaining ground in response to climate change. Since forests play a role as carbon sinks, they are increasingly seen as a key factor in combating climate change, making them part of the global debate on reducing greenhouse gas emissions. 'Good' forest governance from the climate change perspective is more and more driven by multilateral institutions, conventions and coalitions at supranational levels (Van Oosten and Hijweege, 2012).

To govern something in the right way means to get to know it in depth first. Furthermore, it is necessary to quantify it and to identify the factors affecting it. Following Görg (2007), Keen et al. (2005), Leeuwis and Aarts (2010), Massey (2005), Van Paassen et al. (2011), Wals et al. (2009), Wenger (2000) and others, forest learning is an important element of forest governance.

The aforementioned approach has resulted in the formulation of the aim of the paper. The aim is to determine the influence of particular factors on the diversity of the European Union countries in terms of the amount of wood forest resources in relation to the country size. Two factors affecting the variable, namely:

1) the growing stock density, which is the proportion of the volume (over bark) of standing trees to the forest land involved, and

2) the forest cover, which is the proportion of the forest area to the land area of the country (without lands under waters),

3) shall be analysed in this article.

The difference between the value of the studied variable for a given country and the value of this variable for the European Union will be defined as a deviation. Such a deviation may be negative, zero, or positive. Thus, in each case 
the deviation is mentioned in this paper, it shall be assumed as the deviation from the mean EU value. The logarithmic method will be used to assess the influence of the deviations of the said factors on the deviation of the volume of timber forest resources compared with the country size.

\section{RESEARCH METHOD}

The examined variable $\alpha$ (the wood forest resources expressed in $\mathrm{m}^{3}$ per 1 ha of land area) can be presented as a product of factors $\beta$ (the growing stock expressed in $\mathrm{m}^{3}$ per 1 ha of forest area) and $\gamma$ (the extent of forested territory expressed in percent). The value of variable $\alpha$ regarding the entire European Union will be the basis of reference and shall be marked by $\bar{\alpha}$. In turn, the value of this variable calculated for the $i$-th country will be denoted as $\alpha_{i}$. Due to the fact that $\alpha_{i}=\beta_{i} \cdot \gamma_{i}$ and $\bar{\alpha}=\bar{\beta} \cdot \bar{\gamma}$, when dividing $\alpha_{i}$ by $\bar{\alpha}$, the obtained result is:

$$
\frac{\alpha_{i}}{\bar{\alpha}}=\frac{\beta_{i} \cdot \gamma_{i}}{\bar{\beta} \cdot \bar{\gamma}}
$$

where $\alpha_{i}, \beta_{i}, \gamma_{i}$ are the values of variables $\alpha, \beta, \gamma$ referring to the $i$-th country and $\bar{\alpha}, \bar{\beta}, \bar{\gamma}$ are the mean values of variables $\alpha, \beta, \gamma$ referring to the EU. The same can be shown in a different way, namely:

$$
\mathrm{A}_{i}=\mathrm{B}_{i} \cdot \Gamma_{i}
$$

where $\mathrm{A}_{i}=\frac{\alpha_{i}}{\bar{\alpha}}, \mathrm{B}_{i}=\frac{\beta_{i}}{\bar{\beta}}$ and $\Gamma_{i}=\frac{\gamma_{i}}{\bar{\gamma}}$.

From mathematical point of view, logarithms to any base can be taken of both sides of an equation, provided that the numbers that the logarithms have been taken of are positive (Turczak, 2016). The values of ratios $\mathrm{A}_{i}, \mathrm{~B}_{i}$, and $\Gamma_{i}$ are always greater than zero, hence the logarithms can be taken of both sides of the equation (2). The logarithm to the base $e$ will be used in further calculations.

Taking the natural logarithms of both sides of the equation (2), the following expression can be obtained:

$$
\ln \mathrm{A}_{i}=\ln \left(\mathrm{B}_{i} \cdot \Gamma_{i}\right)
$$

Then, using the logarithm property stipulating that the logarithm of a product of some numbers is equal to the sum of the logarithms of these numbers (Turczak, 2017), and then dividing both sides of the equation by the term $\ln \mathrm{A}_{i}$, the equation presented below can be derived:

$$
\frac{\ln \mathrm{A}_{i}}{\ln \mathrm{A}_{i}}=\frac{\ln \mathrm{B}_{i}}{\ln \mathrm{A}_{i}}+\frac{\ln \Gamma_{i}}{\ln \mathrm{A}_{i}}
$$

or shown in a different way:

$$
1=\log _{\mathrm{A}_{i}} \mathrm{~B}_{i}+\log _{\mathrm{A}_{i}} \Gamma_{i}
$$

where $\log _{\mathrm{A}_{i}} \mathrm{~B}_{i}$ and $\log _{\mathrm{A}_{i}} \Gamma_{i}$ are the impacts of the deviations of $\beta$ factor and $\gamma$ factor on the deviation of $\alpha$ variable.

The next step is to multiply both sides of the equation (5) by the value of deviation calculated for variable $\alpha$. This results in the expression:

$$
\alpha_{i}-\bar{\alpha}=\left(\alpha_{i}-\bar{\alpha}\right) \cdot \log _{\mathrm{A}_{i}} \mathrm{~B}_{i}+\left(\alpha_{i}-\bar{\alpha}\right) \cdot \log _{\mathrm{A}_{i}} \Gamma_{i}
$$

where $\alpha_{i}-\bar{\alpha}$ is the total deviation of variable $\alpha$ and $\left(\alpha_{i}-\bar{\alpha}\right) \cdot \log _{\mathrm{A}_{i}} \mathrm{~B}_{i},\left(\alpha_{i}-\bar{\alpha}\right) \cdot \log _{\mathrm{A}_{i}} \Gamma_{i}$ are the deviations of variable $\alpha$ caused by the deviations of factor $\beta$ and factor $\gamma$.

In this article, the causal analysis will allow to answer the question how the said factors influence the deviations of wood forest resources quantities in the twenty eight countries compared to the mean volume characterizing the European Union. The research will be conducted based on data from 2005, 2010 and 2015. 


\section{RESEARCH RESULTS}

\section{Comparing the volume of wood forest resources}

The interesting issue is how the EU Member States vary in terms of the amount of timber forest resources compared with the country size. Table 1 contains the relevant data.

Table 1. The amount of wood forest resources (in $\mathrm{m}^{3} / \mathrm{ha}$ of land area without inland water)

\begin{tabular}{|c|c|c|c|}
\hline Country & 2005 & Country & 2010 \\
\hline Slovenia & 185.7 & Slovenia & 201.7 \\
\hline Austria & 133.7 & Austria & 137.0 \\
\hline Estonia & 104.8 & Estonia & 108.3 \\
\hline Slovakia & 100.9 & Slovakia & 104.8 \\
\hline Germany & 100.4 & Germany & 103.7 \\
\hline Luxembourg & 100.3 & Luxembourg & 100.3 \\
\hline Czech Republic & 95.1 & Latvia & 98.7 \\
\hline Latvia & 89.4 & Czech Republic & 97.7 \\
\hline Lithuania & 74.1 & Lithuania & 78.1 \\
\hline Finland & 71.6 & Poland & 77.5 \\
\hline Sweden & 70.8 & Finland & 76.3 \\
\hline Croatia & 68.0 & Sweden & 71.8 \\
\hline Poland & 62.3 & Croatia & 71.8 \\
\hline Romania & 58.8 & Romania & 59.9 \\
\hline Belgium & 55.8 & Bulgaria & 59.2 \\
\hline EU-28 & 55.1 & Belgium & 58.8 \\
\hline Bulgaria & 54.2 & EU-28 & 58.6 \\
\hline France & 45.7 & France & 48.2 \\
\hline Italy & 39.8 & Italy & 43.4 \\
\hline Hungary & 36.7 & Hungary & 38.6 \\
\hline Denmark & 26.6 & Denmark & 27.5 \\
\hline United Kingdom & 22.1 & United Kingdom & 24.5 \\
\hline Netherlands & 20.7 & Netherlands & 22.5 \\
\hline Spain & 20.5 & Spain & 22.3 \\
\hline Portugal & 20.4 & Portugal & 20.5 \\
\hline Greece & 13.5 & Greece & 14.1 \\
\hline Ireland & 10.1 & Ireland & 13.2 \\
\hline Cyprus & 9.1 & Cyprus & 10.8 \\
\hline Malta & 2.5 & Malta & 2.5 \\
\hline
\end{tabular}

\begin{tabular}{|l|c|}
\hline \multicolumn{1}{|c|}{ Country } & $\mathbf{2 0 1 5}$ \\
\hline Slovenia & 214.2 \\
\hline Austria & 140.2 \\
\hline Estonia & 109.7 \\
\hline Slovakia & 108.5 \\
\hline Latvia & 106.9 \\
\hline Germany & 105.0 \\
\hline Czech Republic & 102.5 \\
\hline Luxembourg & 100.3 \\
\hline Romania & 83.9 \\
\hline Poland & 83.0 \\
\hline Lithuania & 82.2 \\
\hline Finland & 76.3 \\
\hline Sweden & 73.4 \\
\hline Croatia & 73.3 \\
\hline Bulgaria & 64.5 \\
\hline EU-28 & $\mathbf{6 2 . 5}$ \\
\hline Belgium & 61.9 \\
\hline France & 52.0 \\
\hline Italy & 46.9 \\
\hline Hungary & 42.1 \\
\hline Denmark & 29.2 \\
\hline United Kingdom & 26.9 \\
\hline Spain & 24.1 \\
\hline Netherlands & 24.0 \\
\hline Portugal & 20.5 \\
\hline Ireland & 17.1 \\
\hline Greece & 14.8 \\
\hline Cyprus & 12.1 \\
\hline Malta & 2.5 \\
\hline & \\
\hline
\end{tabular}

Source: own computation based on Eurostat database (accessed on 10/11/2017).

The largest quantity of wood forest resources has been recorded in Slovenia - in 2015 it was on average $214.2 \mathrm{~m}^{3}$ of the stock of living trees per each hectare of land surface of the country. Thus, the value was $151.7 \mathrm{~m}^{3}$ larger $(243 \%$ larger) than the mean value obtained for all the discussed countries. In turn, the smallest quantity was observed in Malta - in 2015 the relative measure of timber forest resources in Malta equalled only $2.5 \mathrm{~m}^{3} / \mathrm{ha}$ of land area, i.e. $60.0 \mathrm{~m}^{3}$ less $(96 \%$ less $)$ than the mean volume in the EU.

\section{Comparing the forest growing stock density}

The task is the assessment of the forest growing stock density in each of the studied countries in relation to the mean value in the European Union. All the data needed have been presented in Table 2.

Table 2. The volume of forest growing stock (in $\mathrm{m}^{3} / \mathrm{ha}$ of forest area)

\begin{tabular}{|l|c|}
\hline \multicolumn{1}{|c|}{ Country } & $\mathbf{2 0 0 5}$ \\
\hline Germany & 307.6 \\
\hline Slovenia & 301.0 \\
\hline Luxembourg & 299.1 \\
\hline Austria & 286.2 \\
\hline Czech Republic & 277.7 \\
\hline Slovakia & 256.1 \\
\hline Belgium & 251.1 \\
\hline Malta & 228.6 \\
\hline Lithuania & 219.0 \\
\hline Romania & 211.5 \\
\hline Poland & 207.5 \\
\hline Denmark & 205.0 \\
\hline Croatia & 202.3 \\
\hline Estonia & 202.0 \\
\hline Netherlands & 191.8 \\
\hline
\end{tabular}

\begin{tabular}{|l|l|}
\hline \multicolumn{1}{|c|}{ Country } & $\mathbf{2 0 1 0}$ \\
\hline Slovenia & 325.7 \\
\hline Germany & 317.0 \\
\hline Luxembourg & 299.1 \\
\hline Austria & 292.5 \\
\hline Czech Republic & 284.0 \\
\hline Slovakia & 265.2 \\
\hline Belgium & 262.0 \\
\hline Poland & 254.3 \\
\hline Malta & 228.6 \\
\hline Lithuania & 225.7 \\
\hline Croatia & 211.6 \\
\hline Romania & 211.5 \\
\hline Estonia & 210.5 \\
\hline Netherlands & 203.5 \\
\hline Denmark & 201.0 \\
\hline
\end{tabular}

\begin{tabular}{|l|c|}
\hline \multicolumn{1}{|c|}{ Country } & $\mathbf{2 0 1 5}$ \\
\hline Slovenia & 345.8 \\
\hline Germany & 320.8 \\
\hline Luxembourg & 299.1 \\
\hline Austria & 298.5 \\
\hline Czech Republic & 296.6 \\
\hline Romania & 281.4 \\
\hline Belgium & 274.7 \\
\hline Slovakia & 274.3 \\
\hline Poland & 269.2 \\
\hline Lithuania & 236.2 \\
\hline Malta & 228.6 \\
\hline Croatia & 215.9 \\
\hline Netherlands & 215.2 \\
\hline Estonia & 213.4 \\
\hline United Kingdom & 207.4 \\
\hline
\end{tabular}


Proceedings of the $8^{\text {th }}$ International Scientific Conference Rural Development 2017

\begin{tabular}{|c|c|c|c|}
\hline Country & 2005 & Country & 2010 \\
\hline United Kingdom & 177.8 & United Kingdom & 194.5 \\
\hline Hungary & 172.1 & Latvia & 183.1 \\
\hline Latvia & 168.9 & Hungary & 175.4 \\
\hline Bulgaria & 161.9 & Bulgaria & 172.6 \\
\hline France & 158.4 & France & 161.3 \\
\hline EU-28 & 149.4 & EU-28 & 156.6 \\
\hline Italy & 134.0 & Italy & 141.7 \\
\hline Sweden & 103.0 & Ireland & 124.0 \\
\hline Ireland & 99.8 & Sweden & 105.0 \\
\hline Finland & 98.4 & Finland & 104.4 \\
\hline Spain & 59.4 & Spain & 61.4 \\
\hline Portugal & 56.1 & Portugal & 57.4 \\
\hline Cyprus & 48.5 & Cyprus & 57.4 \\
\hline Greece & 47.2 & Greece & 47.4 \\
\hline
\end{tabular}

\begin{tabular}{|l|c|}
\hline \multicolumn{1}{|c|}{ Country } & $\mathbf{2 0 1 5}$ \\
\hline Denmark & 204.5 \\
\hline Latvia & 198.2 \\
\hline Bulgaria & 182.8 \\
\hline Hungary & 182.2 \\
\hline France & 168.3 \\
\hline EU-28 & $\mathbf{1 6 4 . 9}$ \\
\hline Ireland & 154.9 \\
\hline Italy & 148.9 \\
\hline Sweden & 106.5 \\
\hline Finland & 104.4 \\
\hline Spain & 65.8 \\
\hline Cyprus & 64.4 \\
\hline Portugal & 58.5 \\
\hline Greece & 49.4 \\
\hline
\end{tabular}

The largest amount of growing stock per 1 ha of forest area has been observed in Germany (2005) and in Slovenia (2010 and 2015). In 2015 the value of the measure in Slovenia was more than twice the mean volume in the group of all the twenty eight countries. Greece recorded the lowest forest growing stock density in the examined years - the value of the measure in Greece was about $30 \%$ of the mean value obtained for the EU Member States in total.

\section{Comparing the forest cover}

The next task is to compare the forest area in proportion to the land area (excluding lakes and large rivers) in the studied countries. The necessary data have been given in Table 3.

Table 3. The extent of forested territory (in percent)

\begin{tabular}{|c|c|c|c|}
\hline Country & 2005 & Country & 2010 \\
\hline Finland & 72.8 & Finland & 73.1 \\
\hline Sweden & 68.8 & Sweden & 68.4 \\
\hline Slovenia & 61.7 & Slovenia & 61.9 \\
\hline Latvia & 52.9 & Latvia & 53.9 \\
\hline Estonia & 51.9 & Estonia & 51.4 \\
\hline Austria & 46.7 & Austria & 46.8 \\
\hline Slovakia & 39.4 & Slovakia & 39.5 \\
\hline EU-28 & 36.8 & EU-28 & 37.4 \\
\hline Portugal & 36.3 & Spain & 36.4 \\
\hline Spain & 34.4 & Portugal & 35.7 \\
\hline Czech Republic & 34.3 & Lithuania & 34.6 \\
\hline Lithuania & 33.8 & Czech Republic & 34.4 \\
\hline Croatia & 33.6 & Bulgaria & 34.3 \\
\hline Luxembourg & 33.5 & Croatia & 33.9 \\
\hline Bulgaria & 33.5 & Luxembourg & 33.5 \\
\hline Germany & 32.6 & Germany & 32.7 \\
\hline Poland & 30.0 & Italy & 30.6 \\
\hline Italy & 29.7 & Poland & 30.5 \\
\hline France & 28.8 & France & 29.9 \\
\hline Greece & 28.7 & Greece & 29.8 \\
\hline Romania & 27.8 & Romania & 28.3 \\
\hline Belgium & 22.2 & Belgium & 22.4 \\
\hline Hungary & 21.3 & Hungary & 22.0 \\
\hline Cyprus & 18.8 & Cyprus & 18.8 \\
\hline Denmark & 13.0 & Denmark & 13.7 \\
\hline United Kingdom & 12.5 & United Kingdom & 12.6 \\
\hline Netherlands & 10.8 & Netherlands & 11.1 \\
\hline Ireland & 10.2 & Ireland & 10.6 \\
\hline Malta & 1.1 & Malta & 1.1 \\
\hline
\end{tabular}

\begin{tabular}{|l|c|}
\hline \multicolumn{1}{|c|}{ Country } & $\mathbf{2 0 1 5}$ \\
\hline Finland & 73.1 \\
\hline Sweden & 68.9 \\
\hline Slovenia & 62.0 \\
\hline Latvia & 53.9 \\
\hline Estonia & 51.4 \\
\hline Austria & 46.9 \\
\hline Slovakia & 39.6 \\
\hline EU-28 & $\mathbf{3 7 . 9}$ \\
\hline Spain & 36.7 \\
\hline Bulgaria & 35.3 \\
\hline Portugal & 35.1 \\
\hline Lithuania & 34.8 \\
\hline Czech Republic & 34.5 \\
\hline Croatia & 34.0 \\
\hline Luxembourg & 33.5 \\
\hline Germany & 32.7 \\
\hline Italy & 31.5 \\
\hline France & 30.9 \\
\hline Poland & 30.8 \\
\hline Greece & 29.8 \\
\hline Romania & 29.8 \\
\hline Hungary & 23.1 \\
\hline Belgium & 22.5 \\
\hline Cyprus & 18.7 \\
\hline Denmark & 14.3 \\
\hline United Kingdom & 13.0 \\
\hline Netherlands & 11.2 \\
\hline Ireland & 11.0 \\
\hline Malta & 1.1 \\
\hline
\end{tabular}

In the examined years, the biggest share of the forest area in the land area was noted in Finland - nearly three quarters of the land territory was forested in this country. The smallest share of the forested surface in the land area was observed in the case of Malta - in those years the level of the measure in Malta was about thirty four times lower than the mean value obtained for all the discussed countries.

\section{Computing the impacts and impact effects of the two factors}


The last task to be carried out is the evaluation of the influence of deviations of the analysed factors on the deviations of the wood forest resources quantities in relation to land territories in the given countries.

It was established in this paper that the value of the dependent variable $(\alpha)$ may be calculated by multiplication of 1 ) the living stock of standing wood per 1 ha of forest area $(\beta)$ and 2) the quotient of the forest area and the land area $(\gamma)$. The (2) ratio equality was derived from this relationship.

In the last part of this research the remaining stages of the logarithmic method will be performed. This will result in receiving information regarding the impact effect of the first factor and the impact effect of the second factor on the deviation of the dependent variable. The results obtained for 2005, 2010, and 2015 are shown in Table 4.

Table 4. The occurring deviations of variable $\alpha$ and the causes of the deviations

\begin{tabular}{|c|c|c|c|c|}
\hline Country & $1^{\circ} / 2^{\circ}$ & 2005 & 2010 & 2015 \\
\hline $\mathrm{B}_{i}>\mathbf{1}, \Gamma_{i}>\mathbf{1} \quad\left(\alpha_{i}-\bar{c}\right.$ & \multicolumn{4}{|c|}{$\mathrm{B}_{i}>\mathbf{1}, \Gamma_{i}>\mathbf{1} \quad\left(\alpha_{i}-i\right.$} \\
\hline Slovenia & $\begin{array}{l}1^{\circ} \\
2^{\circ}\end{array}$ & $\begin{aligned} \mathbf{3 . 3 7 4} & =2.014 \cdot 1.675 \\
(+\mathbf{1 3 0 . 7}) & =(+75.3)+(+55.4)\end{aligned}$ & $\begin{aligned} \mathbf{3 . 4 4 0} & =2.080 \cdot 1.654 \\
(+\mathbf{1 4 3 . 0}) & =(+84.8)+(+58.3)\end{aligned}$ & $\begin{aligned} \mathbf{3 . 4 2 9} & =2.097 \cdot 1.635 \\
(+\mathbf{1 5 1 . 7}) & =(+91.2)+(+60.5)\end{aligned}$ \\
\hline Austria & $\begin{array}{l}1^{\circ} \\
2^{\circ}\end{array}$ & $\begin{array}{c}\mathbf{2 . 4 2 8}=1.915 \cdot 1.268 \\
(+\mathbf{7 8 . 6})=(+57.6)+(+21.0)\end{array}$ & $\begin{array}{c}\mathbf{2 . 3 3 7}=1.868 \cdot 1.251 \\
(+\mathbf{7 8 . 4})=(+57.7)+(+20.7)\end{array}$ & $\begin{array}{c}\mathbf{2 . 2 4 3}=1.811 \cdot 1.239 \\
(+\mathbf{7 7 . 7})=(+57.1)+(+20.6)\end{array}$ \\
\hline Estonia & $\begin{array}{l}1^{\circ} \\
2^{\circ}\end{array}$ & $\begin{array}{c}\mathbf{1 . 9 0 3}=1.352 \cdot 1.407 \\
(+\mathbf{4 9 . 7})=(+23.3)+(+26.4)\end{array}$ & $\begin{array}{c}\mathbf{1 . 8 4 7}=1.344 \cdot 1.374 \\
(+\mathbf{4 9 . 7})=(+24.0)+(+25.7)\end{array}$ & $\begin{array}{c}\mathbf{1 . 7 5 5}=1.294 \cdot 1.356 \\
(+\mathbf{4 7 . 2})=(+21.6)+(+25.6)\end{array}$ \\
\hline Slovakia & $\begin{array}{l}1^{\circ} \\
2^{\circ}\end{array}$ & $\begin{aligned} \mathbf{1 . 8 3 2} & =1.714 \cdot 1.069 \\
(+\mathbf{4 5 . 8}) & =(+40.8)+(+5.1)\end{aligned}$ & $\begin{array}{c}\mathbf{1 . 7 8 8}=1.693 \cdot 1.056 \\
(+\mathbf{4 6 . 2})=(+41.9)+(+4.3)\end{array}$ & $\begin{array}{c}\mathbf{1 . 7 3 7}=1.663 \cdot 1.044 \\
(+\mathbf{4 6 . 0})=(+42.4)+(+3.6)\end{array}$ \\
\hline Latvia & $\begin{array}{l}1^{\circ} \\
2^{\circ}\end{array}$ & $\begin{aligned} \mathbf{1 . 6 2 4} & =1.131 \cdot 1.436 \\
(+\mathbf{3 4 . 4}) & =(+8.7)+(+25.7)\end{aligned}$ & $\begin{array}{c}\mathbf{1 . 6 8 4}=1.169 \cdot 1.440 \\
(+\mathbf{4 0 . 1})=(+12.0)+(+28.1)\end{array}$ & $\begin{array}{c}\mathbf{1 . 7 1 1}=1.202 \cdot 1.424 \\
(+\mathbf{4 4 . 4})=(+15.2)+(+29.2)\end{array}$ \\
\hline \multicolumn{5}{|c|}{$\mathrm{B}_{i}>\mathbf{1}, \Gamma_{i}<\mathbf{1} \quad\left(\alpha_{i}-\bar{\alpha}\right) \cdot \log _{\mathrm{A}_{i}} \mathrm{~B}_{i}>\mathbf{0},\left(\alpha_{i}-\bar{\alpha}\right) \cdot \log _{\mathrm{A}_{i}} \Gamma_{i}<\mathbf{0}$} \\
\hline Germany & $\begin{array}{l}1^{\circ} \\
2^{\circ}\end{array}$ & $\begin{aligned} \mathbf{1 . 8 2 4} & =2.059 \cdot 0.886 \\
(+\mathbf{4 5 . 4}) & =(+54.5)+(-9.2)\end{aligned}$ & $\begin{array}{c}\mathbf{1 . 7 6 9}=2.025 \cdot 0.874 \\
(+\mathbf{4 5 . 1})=(+55.7)+(-10.7)\end{array}$ & $\begin{array}{c}\mathbf{1 . 6 8 1}=1.946 \cdot 0.864 \\
(+42.5)=(+54.5)+(-12.0)\end{array}$ \\
\hline $\begin{array}{l}\text { Czech } \\
\text { Republic }\end{array}$ & $\begin{array}{l}1^{\circ} \\
2^{\circ}\end{array}$ & $\begin{aligned} \mathbf{1 . 7 2 8} & =1.858 \cdot 0.930 \\
(+\mathbf{4 0 . 1}) & =(+45.4)+(-5.3)\end{aligned}$ & $\begin{aligned} \mathbf{1 . 6 6 7} & =1.813 \cdot 0.919 \\
(+\mathbf{3 9 . 1}) & =(+45.5)+(-6.5)\end{aligned}$ & $\begin{array}{c}\mathbf{1 . 6 4 0}=1.799 \cdot 0.912 \\
(+40.0)=(+47.5)+(-7.5)\end{array}$ \\
\hline Luxemburg & $\begin{array}{l}1^{\circ} \\
2^{\circ}\end{array}$ & $\begin{aligned} \mathbf{1 . 8 2 3} & =2.002 \cdot 0.910 \\
(+\mathbf{4 5 . 3}) & =(+52.4)+(-7.1)\end{aligned}$ & $\begin{aligned} \mathbf{1 . 7 1 2} & =1.910 \cdot 0.896 \\
(+\mathbf{4 1 . 7}) & =(+50.2)+(-8.5)\end{aligned}$ & $\begin{array}{c}\mathbf{1 . 6 0 6}=1.814 \cdot 0.885 \\
(+37.9)=(+47.6)+(-9.7)\end{array}$ \\
\hline Romania & $\begin{array}{l}1^{\circ} \\
2^{\circ}\end{array}$ & $\begin{array}{c}\mathbf{1 . 0 6 8}=1.416 \cdot 0.754 \\
(+\mathbf{+ 3 . 7})=(+19.8)+(-16.1)\end{array}$ & $\begin{array}{c}\mathbf{1 . 0 2 2}=1.351 \cdot 0.756 \\
(+\mathbf{1 . 3})=(+17.8)+(-16.5)\end{array}$ & $\begin{array}{c}\mathbf{1 . 3 4 3}=1.706 \cdot 0.787 \\
(+21.4)=(+38.8)+(-17.4)\end{array}$ \\
\hline Poland & $\begin{array}{l}1^{\circ} \\
2^{\circ}\end{array}$ & $\begin{array}{c}\mathbf{1 . 1 3 3}=1.389 \cdot 0.815 \\
(+\mathbf{7 . 3})=(+19.3)+(-12.0)\end{array}$ & $\begin{array}{c}\mathbf{1 . 3 2 1}=1.624 \cdot 0.814 \\
(+\mathbf{1 8 . 8})=(+32.8)+(-13.9)\end{array}$ & $\begin{array}{c}\mathbf{1 . 3 2 8}=1.633 \cdot 0.813 \\
(+\mathbf{2 0 . 5})=(+35.4)+(-14.9)\end{array}$ \\
\hline Lithuania & $\begin{array}{l}1^{\circ} \\
2^{\circ}\end{array}$ & $\begin{aligned} \mathbf{1 . 3 4 6} & =1.466 \cdot 0.918 \\
(+\mathbf{1 9 . 1}) & =(+24.5)+(-5.5)\end{aligned}$ & $\begin{array}{c}\mathbf{1 . 3 3 3}=1.441 \cdot 0.925 \\
(+\mathbf{1 9 . 5})=(+24.8)+(-5.3)\end{array}$ & $\begin{array}{c}\mathbf{1 . 3 1 5}=1.433 \cdot 0.918 \\
(+\mathbf{1 9 . 7})=(+25.9)+(-6.1)\end{array}$ \\
\hline Croatia & $\begin{array}{l}1^{\circ} \\
2^{\circ}\end{array}$ & $\begin{aligned} \mathbf{1 . 2 3 6} & =1.354 \cdot 0.913 \\
(+\mathbf{1 3 . 0}) & =(+18.6)+(-5.6)\end{aligned}$ & $\begin{array}{c}\mathbf{1 . 2 2 5}=1.351 \cdot 0.906 \\
(+\mathbf{1 3 . 2})=(+19.6)+(-6.4)\end{array}$ & $\begin{array}{c}\mathbf{1 . 1 7 3}=1.309 \cdot 0.896 \\
(+\mathbf{1 0 . 8})=(+18.3)+(-7.4)\end{array}$ \\
\hline Bulgaria & $\begin{array}{l}1^{\circ} \\
2^{\circ}\end{array}$ & $\begin{array}{c}0.985=1.083 \cdot 0.909 \\
(-0.8)=(+4.4)+(-5.2)\end{array}$ & $\begin{array}{c}\mathbf{1 . 0 1 0}=1.102 \cdot 0.916 \\
(+\mathbf{0 . 6})=(+5.7)+(-5.2)\end{array}$ & $\begin{array}{c}\mathbf{1 . 0 3 2}=1.109 \cdot 0.931 \\
(+\mathbf{2 . 0})=(+6.6)+(-4.6)\end{array}$ \\
\hline Belgium & $\begin{array}{l}1^{\circ} \\
2^{\circ}\end{array}$ & $\begin{array}{c}\mathbf{1 . 0 1 4}=1.681 \cdot 0.603 \\
(+\mathbf{0 . 8})=(+28.8)+(-28.0)\end{array}$ & $\begin{array}{c}\mathbf{1 . 0 0 2}=1.673 \cdot 0.599 \\
(+\mathbf{0 . 1})=(+30.2)+(-30.1)\end{array}$ & $\begin{array}{c}0.991=1.666 \cdot 0.595 \\
(-0.6)=(+31.7)+(-32.3)\end{array}$ \\
\hline France & $\begin{array}{l}1^{\circ} \\
2^{\circ}\end{array}$ & $\begin{array}{c}0.829=1.060 \cdot 0.782 \\
(-9.4)=(+2.9)+(-12.3)\end{array}$ & $\begin{array}{c}0.821=1.030 \cdot 0.798 \\
(-10.5)=(+1.6)+(-12.0)\end{array}$ & $\begin{array}{c}0.832=1.021 \cdot 0.815 \\
(-10.5)=(+1.2)+(-11.7)\end{array}$ \\
\hline Hungary & $\begin{array}{l}1^{\circ} \\
2^{\circ}\end{array}$ & $\begin{array}{c}0.667=1.152 \cdot 0.579 \\
(-18.4)=(+6.4)+(-24.8)\end{array}$ & $\begin{array}{c}0.658=1.120 \cdot 0.588 \\
(-20.0)=(+5.4)+(-25.5)\end{array}$ & $\begin{array}{c}0.673=1.105 \cdot 0.609 \\
(-20.4)=(+5.2)+(-25.6)\end{array}$ \\
\hline Denmark & $\begin{array}{l}1^{\circ} \\
2^{\circ}\end{array}$ & $\begin{array}{c}0.484=1.372 \cdot 0.353 \\
(-28.4)=(+12.4)+(-40.8)\end{array}$ & $\begin{array}{c}0.469=1.284 \cdot 0.365 \\
(-31.1)=(+10.3)+(-41.4)\end{array}$ & $\begin{array}{c}0.467=1.240 \cdot 0.376 \\
(-33.3)=(+9.4)+(-42.7)\end{array}$ \\
\hline $\begin{array}{l}\text { United } \\
\text { Kingdom }\end{array}$ & $\begin{array}{l}1^{\circ} \\
2^{\circ}\end{array}$ & $\begin{array}{c}0.402=1.190 \cdot 0.338 \\
(-32.9)=(+6.3)+(-39.2)\end{array}$ & $\begin{array}{c}0.419=1.242 \cdot 0.337 \\
(-34.1)=(+8.5)+(-42.6)\end{array}$ & $\begin{array}{c}0.430=1.258 \cdot 0.342 \\
(-35.6)=(+9.7)+(-45.3)\end{array}$ \\
\hline Netherlands & $\begin{array}{l}1^{\circ} \\
2^{\circ}\end{array}$ & $\begin{array}{c}0.376=1.284 \cdot 0.293 \\
(-34.3)=(+8.8)+(-43.1)\end{array}$ & $\begin{array}{c}0.384=1.300 \cdot 0.296 \\
(-36.1)=(+9.9)+(-46.0)\end{array}$ & $\begin{array}{c}0.384=1.305 \cdot 0.295 \\
(-38.5)=(+10.7)+(-49.2)\end{array}$ \\
\hline Malta & $\begin{array}{l}1^{\circ} \\
2^{\circ}\end{array}$ & $\begin{aligned} 0.046 & =1.530 \cdot 0.030 \\
(-52.5) & =(+7.3)+(-59.8)\end{aligned}$ & $\begin{array}{c}0.043=1.460 \cdot 0.030 \\
(-56.1)=(+6.8)+(-62.8)\end{array}$ & $\begin{array}{c}0.041=1.386 \cdot 0.029 \\
(-60.0)=(+6.1)+(-66.1)\end{array}$ \\
\hline \multicolumn{5}{|c|}{$\mathrm{B}_{i}<\mathbf{1}, \Gamma_{i}>\mathbf{1} \quad\left(\alpha_{i}-\bar{\alpha}\right) \cdot \log _{\mathrm{A}_{i}} \mathrm{~B}_{i}<\mathbf{0},\left(\alpha_{i}-\bar{\alpha}\right) \cdot \log _{\mathrm{A}_{i}} \Gamma_{i}>\mathbf{0}$} \\
\hline Finland & $\begin{array}{l}1^{\circ} \\
2^{\circ}\end{array}$ & $\begin{array}{c}\mathbf{1 . 3 0 1}=0.659 \cdot 1.975 \\
(+\mathbf{1 6 . 6})=(-26.3)+(+42.9)\end{array}$ & $\begin{array}{c}\mathbf{1 . 3 0 2}=0.667 \cdot 1.953 \\
(+\mathbf{1 7 . 7})=(-27.2)+(+44.9)\end{array}$ & $\begin{array}{c}\mathbf{1 . 2 2 2}=0.633 \cdot 1.929 \\
(+\mathbf{1 3 . 9})=(-31.6)+(+45.5)\end{array}$ \\
\hline Sweden & $\begin{array}{l}1^{\circ} \\
2^{\circ}\end{array}$ & $\begin{array}{c}\mathbf{1 . 2 8 7}=0.690 \cdot 1.866 \\
(+\mathbf{1 5 . 8})=(-23.3)+(+39.1)\end{array}$ & $\begin{array}{c}\mathbf{1 . 2 2 5}=0.671 \cdot 1.828 \\
(+\mathbf{1 3 . 2})=(-26.0)+(+39.2)\end{array}$ & $\begin{array}{c}\mathbf{1 . 1 7 4}=0.646 \cdot 1.819 \\
(+\mathbf{1 0 . 9})=(-29.7)+(+40.5)\end{array}$ \\
\hline \multicolumn{5}{|c|}{$\mathrm{B}_{i}<\mathbf{1}, \Gamma_{i}<\mathbf{1} \quad\left(\alpha_{i}-\bar{\alpha}\right) \cdot \log _{\mathrm{A}_{i}} \mathrm{~B}_{i}<\mathbf{0},\left(\alpha_{i}-\bar{\alpha}\right) \cdot \log _{\mathrm{A}_{i}} \Gamma_{i}<\mathbf{0}$} \\
\hline Italy & $\begin{array}{l}1^{\circ} \\
2^{\circ} \\
\end{array}$ & $\begin{array}{c}0.723=0.897 \cdot 0.805 \\
(-15.3)=(-5.1)+(-10.2)\end{array}$ & $\begin{array}{c}0.740=0.905 \cdot 0.817 \\
(-15.3)=(-5.1)+(-10.2)\end{array}$ & $\begin{aligned} 0.751 & =0.903 \cdot 0.831 \\
(-15.6) & =(-5.5)+(-10.0)\end{aligned}$ \\
\hline Spain & $1^{\circ}$ & $0.372=0.398 \cdot 0.935$ & $0.381=0.392 \cdot 0.971$ & $0.386=0.399 \cdot 0.968$ \\
\hline
\end{tabular}




\begin{tabular}{|l|c|c|c|c|}
\hline Country & $1^{\circ} / 2^{\circ}$ & $\mathbf{2 0 0 5}$ & $\mathbf{2 0 1 0}$ & $\mathbf{2 0 1 5}$ \\
\hline & $2^{\circ}$ & $(-34.6)=(-32.2)+(-2.4)$ & $(-36.3)=(-35.2)+(-1.1)$ & $(-38.4)=(-37.0)+(-1.3)$ \\
\hline \multirow{2}{*}{ Portugal } & $1^{\circ}$ & $0.371=0.376 \cdot 0.986$ & $0.350=0.367 \cdot 0.954$ & $0.328=0.355 \cdot 0.926$ \\
& $2^{\circ}$ & $(-34.7)=(-34.2)+(-0.5)$ & $(-38.1)=(-36.4)+(-1.7)$ & $(-42.0)=(-39.1)+(-2.9)$ \\
\hline \multirow{2}{*}{ Ireland } & $1^{\circ}$ & $0.184=0.668 \cdot 0.276$ & $0.224=0.792 \cdot 0.283$ & $0.273=0.940 \cdot 0.291$ \\
& $2^{\circ}$ & $(-44.9)=(-10.7)+(-34.2)$ & $(-45.5)=(-7.1)+(-38.4)$ & $(-45.4)=(-2.2)+(-43.2)$ \\
\hline \multirow{2}{*}{ Greece } & $1^{\circ}$ & $0.246=0.316 \cdot 0.778$ & $0.241=0.303 \cdot 0.797$ & $0.236=0.300 \cdot 0.787$ \\
& $2^{\circ}$ & $(-41.5)=(-34.1)+(-7.4)$ & $(-44.5)=(-37.4)+(-7.1)$ & $(-47.7)=(-39.8)+(-7.9)$ \\
\hline \multirow{2}{*}{ Cyprus } & $1^{\circ}$ & $0.165=0.325 \cdot 0.509$ & $0.184=0.366 \cdot 0.501$ & $0.193=0.391 \cdot 0.495$ \\
& $2^{\circ}$ & $(-46.0)=(-28.7)+(-17.2)$ & $(-47.9)=(-28.3)+(-19.5)$ & $(-50.4)=(-28.8)+(-21.6)$ \\
\hline
\end{tabular}

$1^{\circ}$ - the ratio equality: $\mathrm{A}_{i}=\mathrm{B}_{i} \cdot \Gamma_{i}$

Source: own computation based on Tables $1-3$.

As an example, the values obtained for Lithuania shall be interpreted. In 2015 in Lithuania the amount of wood forest resources was $82.2 \mathrm{~m}^{3}$ per 1 ha of land area and in the EU $-62.5 \mathrm{~m}^{3}$. Thus, in Lithuania it was $19.7 \mathrm{~m}^{3}$ per each ha of land territory greater (i.e. $31.5 \%$ greater) than the mean value computed for the EU. The difference between the value of the measure observed in Lithuania and the analogous value calculated for the group of twenty eight countries taken together was due to the following causes:

- the volume of living standing stock per 1 ha of forest area was $43.3 \%$ higher $\left(236.2 \mathrm{~m}^{3} / \mathrm{ha}\right.$ against $\left.164.9 \mathrm{~m}^{3} / \mathrm{ha}\right)$, and

- the forest cover in Lithuania was lower than in the entire European Union - it was approximately $1 / 10$ lower (34.8 percent versus 37.9 percent).

If the growing stock density had been in Lithuania at the EU level, the amount of timber forest resources in Lithuania would have been $6.1 \mathrm{~m}^{3}$ per each ha of land area smaller than the EU mean volume, what would have been a result solely of the lower forest cover. However, had Lithuania had the forest area in proportion to the land surface the same as it was on average in the EU countries, the volume of wood forest resources in Lithuania would have been 25.9 $\mathrm{m}^{3}$ per each ha of land territory greater than the EU mean volume, only due to the higher growing stock density.

\section{CONCLUSIONS}

Forests play a fundamental role in combating rural poverty, ensuring food security and providing decent livelihoods. They deliver vital long-term ecosystem services, such as clean air and water, conservation of biodiversity and mitigation of climate change (Global..., 2016).

The European Union accounts for approximately 5\% of the world's forests and, contrary to what is happening in many other parts of the world, the forested area of the EU is slowly increasing. Socio-economically, European forests vary from small family holdings to state forests or to large estates owned by companies (Forests..., 2017).

In 2015 the EU-28 had close to 161 million hectares of forests, corresponding to $37.9 \%$ of its land area. The growing stock of timber in forests in the EU-28 totalled some 26.5 billion $\mathrm{m}^{3}$. The task of assessing the volume of wood forest resources in individual European Union countries against the mean quantity characterizing the EU as a whole was carried out in this paper. The growing stock density and the forest cover have been adopted as the factors affecting the said variable. The causal analysis was conducted, enabling the examination of the structure of the deviations of the wood forest resources volumes in the EU Member States.

Finally, it is worth emphasizing that forests not only provide valuable timber, but also a large variety of non-timber forest products, such as food, fodder, medicines, construction materials and tools (Belcher et al., 2005; Neumann and Hirsch, 2000). These products comprise plant and animal products (Ros-Tonen, 2000). A large number of studies and reviews (e.g. Kusters et al., 2006; Ros-Tonen and Wiersum, 2005; Vedeld et al., 2007) provide insight into how nontimber forest products are used worldwide and - what is interesting - the use patterns are remarkably similar across the world. Non-timber forest products provide input to a wide range of industries, including food and beverages, pharmaceuticals, cosmetics and botanical medicines (Ros-Tonen, 2012). Thus, in the following studies, the author is going to investigate the diversity of the European Union countries in terms of the non-timber forest resources.

\section{REFERENCES}

1. Arts, B., Van Bommel, S., Ros-Tonen, M., Verschoor, G. 2012. Forest-people interfaces: from local creativity to global concerns. In: Arts, B., Van Bommel, S., Ros-Tonen, M., Verschoor, G. (eds.), Forest-people interfaces. Understanding community forestry and biocultural diversity. Wageningen Academic Publishers, Wageningen, the Netherlands, pp. 15-26. https://doi.org/10.3920/978-90-8686-749-3_1

2. Arts, B., Visseren-Hamakers, I. 2012. Forest governance: a state of the art review. In: Arts, B., Van Bommel, S., Ros-Tonen, M., Verschoor, G. (eds.), Forest-people interfaces. Understanding community forestry and biocultural diversity. Wageningen Academic Publishers, Wageningen, the Netherlands, pp. 241-257. https://doi.org/10.3920/978-90-8686-749-3

3. Belcher, B., Ruiz-Pérez, M., Achdiawan, R. 2005. Global patterns and trends in the use and management of commercial NTFPs: implications for livelihoods and conservation. World Development, Vol. 33, pp. $1435-1452$. https://doi.org/10.1016/j.worlddev.2004.10.007 


\section{Proceedings of the $8^{\text {th }}$ International Scientific Conference Rural Development 2017}

4. Bevir, M. 2010. Democratic governance. Princeton University Press, Princeton, NJ, USA. https://doi.org/10.1515/9781400836857

5. Food and Agriculture Organization of the United Nations. 2016. Global Forest Resources Assessment 2015. How are the world's forests changing? Second edition.

6. Forests, forestry and logging. 2017. Available at: http://ec.europa.eu/eurostat/statisticsexplained/index.php/Forests,_forestry_and_logging (Accessed on 10/11/2017).

7. Görg, C. 2007. Landscape governance - The 'politics of scale' and the 'natural' conditions of places. Geoforum 38, pp. 954-966. https://doi.org/10.1016/j.geoforum.2007.01.004

8. Held, D., McGrew, A. 2002. Governing globalization: power, authority and global governance. Polity Press, Cambridge, UK.

9. Hooghe, L., Marks, G. 2001. Multi-level governance and European integration. Rowman and Littlefield, Lanham, MD, USA.

10. Keen, M., Brown, V.A., Dyball, R. 2005. Social learning in environmental management - Towards a sustainable future. EarthScan, London, UK.

11. Kjaer, A. 2004. Governance. Polity Press, Cambridge, UK.

12. Kusters, K., Achdiawan, R., Belcher, B., Ruiz-Pérez, M. 2006. Balancing development and conservation? An assessment of livelihood and environmental outcomes of nontimber forest product trade in Asia, Africa, and Latin America. Ecology and Society, Vol. 11(2). https://www.ecologyandsociety.org/vol11/iss2/art20/ (Accessed on 10/11/2017).

13. Leeuwis, C., Aarts, N. 2010. Rethinking communication in innovation processes: creating space for change in complex systems. Paper presented at the 9th European IFSA Symposium, 4-7 July 2010, Vienna, Austria.

14. Massey, D.B. 2005. Landscape as a provocation: reflections on moving mountains. Journal of Material Culture, Vol. 11, pp. 33-48. https://doi.org/10.1177/1359183506062991

15. Neumann, R.P., Hirsch, E. 2000. Commercialisation of non-timber forest products: Review and analysis of research. Center for International Forestry Research, Bogor, Indonesia.

16. Pierre, J. 2000. Debating governance authority, steering and democracy. Oxford University Press, Oxford, UK.

17. Pierre, J., Peters, G. 2000. Governance, politics and the state. Macmillan, London, UK.

18. Ros-Tonen, M.A.F. 2000. The role of non-timber forest products in sustainable tropical forest management. Holz als Roh- und Werkstoff, Vol. 58, pp. 196-201. https://doi.org/10.1007/s001070050413

19. Ros-Tonen, M.A.F. 2012. Non-timber forest product extraction as a productive bricolage process. In: Arts, B., Van Bommel, S., Ros-Tonen, M., Verschoor, G. (eds.), Forest-people interfaces. Understanding community forestry and biocultural diversity. Wageningen Academic Publishers, Wageningen, the Netherlands, pp. 29-48. https://doi.org/10.3920/978-90-8686-749-3_2

20. Ros-Tonen, M.A.F., Wiersum, K.F. 2005. The scope of improving rural livelihood through non-timber forest products: an evolving research agenda. Forest, Trees and Livelihoods, Vol. 15, pp. 129-148. https://doi.org/10.1080/14728028.2005.9752516

21. Turczak, A. 2016. Differences in tourism receipts between Mexico and other countries. Estudios en Ciencias Sociales y Administrativas de la Universidad de Celaya, No. 6, pp. 65-81.

22. Turczak, A. 2017. Characteristics of saving generated by the Baltic countries. Messenger of Armenian State University of Economics, Vol. 2, pp. 80-92.

23. Van Oosten, C.J., Hijweege, W.L. 2012. Governing biocultural diversity in mosaic landscapes. In: Arts, B., Van Bommel, S., RosTonen, M., Verschoor, G. (eds.), Forest-people interfaces. Understanding community forestry and biocultural diversity. Wageningen Academic Publishers, Wageningen, the Netherlands, pp. 211-222. https://doi.org/10.3920/978-90-8686-749-3_13

24. Van Paassen, A., Van den Berg, J., Steingröver, E., Werkman, R., Pedroli, B. 2011. Knowledge in action - The search for collaborative research in sustainable landscape development. Mansholt Publication Series, No. 11, Wageningen Academic Publishers, Wageningen, the Netherlands. https://doi.org/10.3920/978-90-8686-724-0

25. Vedeld, P., Angelsen, A., Bojö, J., Sjaastad, E., Berg, G.K. 2007. Forest environmental incomes and the rural poor. Forest Policy and Economics, Vol. 9, pp. 869-879. https://doi.org/10.1016/j.forpol.2006.05.008

26. Wals, A.E.J., Van der Hoeven, N., Blanken, H. 2009. The acoustics of social learning: designing learning processes that contribute to a more sustainable world. SenterNovem, Utrecht, the Netherlands.

27. Wenger, E.C. 2000. Communities of practice and social learning systems. Organisation, Vol. 7, pp. $225-246$. https://doi.org/10.1177/135050840072002 